\title{
躍Iresalce
}

\section{Plataforma Moodlecloud na qualificação de profissionais de saúde no processo de adesão à Farmacoterapia}

\author{
Moodlecloud Platform in the Qualification of Health Professionals in the Process of \\ Adherence to Pharmacotherapy
}

Marcelo Rodrigues Reser ${ }^{1}$, Clécio Homrich da Silva²

\begin{abstract}
Resumo
Introdução: Elevado consumo, falta de prescrições e de informações podem causar problemas relacionados ao uso de medicamentos. A concordância com a farmacoterapia prescrita (adesão) é fundamental para o paciente alcançar metas terapêuticas estabelecidas. Objetivo: desenvolver um curso para qualificar os profissionais de saúde na promoção da adesão dos pacientes à farmacoterapia por intermédio de um ambiente virtual de aprendizagem. Método: estudo prospectivo e descritivo no desenvolvimento de um curso, na plataforma Moodlecloud, utilizando proposta sócio-construtivista para abordar o tema e estratégia da educação permanente em saúde (reflexão crítica). Participantes avaliaram o curso. Resultados e discussão: na autoavaliação pré-curso, os participantes relataram variável compreensão sobre adesão à farmacoterapia e fatores que a influenciam e baixa abordagem do tema da adesão junto ao paciente. O curso proporcionou espaço para debate de forma crítica, aproximando-se da proposta da educação permanente em saúde. O uso de tecnologia facilitou os contatos assíncronos e à distância. Conclusão: o curso permitiu troca de experiências e construção do conhecimento. As avaliações aperfeiçoaram as atividades. O envolvimento de teoria, prática e experiências valorizaram o curso, favorecendo sua inserção como uma prática em educação permanente em saúde.
\end{abstract}

Palavras-chave: adesão à medicação, educação em saúde, educação a distância.

\section{Abstract}

Introduction: High consumption of medicines, lack of prescriptions and information can cause problems related to the use of medicines. Concordance with the prescribed pharmacotherapy (adherence) is fundamental for the patient to reach established therapeutic goals. Objective: to develop a course to qualify health professionals in promoting patients' adherence to pharmacotherapy through a virtual learning environment. Method: a prospective and descriptive study in the development of a course in the Moodlecloud plataform using socio-constructivist proposal to approach the theme and strategy of the permanent education in health (critical reflection). Participants evaluated the course. Results and discussion: in the pre-course self-assessment, participants reported a variable understanding about adherence to pharmacotherapy and factors that influence it and low approach to the subject of adherence with the patient. The course provided a space for debate in a critical way, approaching the proposal of permanent education in health. The use

1 Mestre. Universidade Federal do Rio Grande do Sul. Email: reserbr@yahoo.com.br

2 Doutor. Hospital de Clínicas de Porto Alegre / Universidade Federal do Rio Grande do Sul. Email: clecio.homrich@ufrgs.br Correspondência: UFRS, Faculdade de Medicina - Rua Ramiro Barcelos, 2400. Santa Cecília. Porto Alegre, RS - Brasil. CEP: 90035003. 
of technology facilitated asynchronous and distance contacts. Conclusion: the course allowed the exchange of experiences and knowledge construction. The evaluations improved the activities. The involvement of theory, practice and experiences valued the course favoring its insertion as a practice in permanent education in health.

Keywords: medication adherence, health education, education distance.

\section{Introdução}

Os medicamentos são insumos essenciais em saúde e determinantes para um melhor desfecho no tratamento de grande número de doenças e agravos à saúde, contribuindo para o aumento da sobrevida e alívio do sofrimento ${ }^{1}$. Eles são considerados relevantes para a proteção e a recuperação da saúde e satisfazem às necessidades de saúde prioritárias da população, devendo estar acessíveis em todos os momentos, na dose apropriada, a todos os segmentos da sociedade ${ }^{2}$.

O consumo de medicamentos, além das indicações terapêuticas, é condicionado por fatores sócioantropológicos, comportamentais e econômicos. A oferta de produtos farmacêuticos, a publicidade e a propaganda criada pela indústria farmacêutica, a quantidade de medicamentos prescritos, as atitudes culturais e também os aumentos da expectativa de vida da população e da prevalência dos transtornos de humor e das doenças resultantes da degradação do meio ambiente, colaboram como estímulos para práticas irracionais dos usos de medicamentos ${ }^{3}$.
Um aspecto importante que prejudica 0 alcance dos resultados pretendidos com a terapia medicamentosa (TM) é a não adesão ao tratamento apresentado. A falta de adesão ao tratamento pelo paciente é considerada como um problema de saúde pública ${ }^{4}$ tem sido denominada de "epidemia invisível" e pode acarretar em falha terapêutica, e em situação extrema, levar ao óbito ${ }^{5}$. Outras situações que podem resultar em sequelas temporárias ou permanentes e casos fatais causados pelo uso inadequado de medicamentos são as intoxicações ${ }^{6}$, reações $\quad$ adversas $^{7 ; 8}$, interações medicamentosas ${ }^{9}$, perda de eficácia, como a resistência a antimicrobianos ${ }^{10}$ e desenvolvimento ou manutenção de dependência de medicamentos e outras substâncias $^{11}$

Para a Organização Mundial da Saúde (OMS), a adesão à terapia de longo prazo é definida como o comportamento de uma pessoa - ou seja, tomar medicação, seguir uma dieta e/ou executar mudanças de estilo de vida correspondentes às recomendações recebidas de um profissional de saúde ${ }^{12}$.

A política que envolve a promoção da saúde propõe a melhora da qualidade 
de vida e a redução da vulnerabilidade e dos riscos relacionados aos determinantes e condicionantes sociais da saúde, e tem como uma de suas estratégias a qualificação dos profissionais de saúde ${ }^{13}$.

Ao refletir sobre a promoção da saúde, alguns desafios se destacam, tais como: tentativa de superar questões institucionais limitadoras, reorientar as práticas profissionais para o acesso à saúde, apresentar qualidade e atenção integral à saúde e incentivar a participação social no acompanhamento e controle das políticas de saúde, fortalecendo a cidadania, a autonomia e o empoderamento individual.

Também não se pode deixar de ressaltar que a educação pode ser considerada uma ferramenta poderosa para o desenvolvimento da promoção da saúde. Mais do que apenas transmissão de informação, a educação em saúde permite a prática do ouvir, a criação de vínculos entre indivíduo e profissional ou equipe, a compreensão de que culturas individuais e sociais influenciam na tomada de decisão sobre a saúde de cada pessoa, o conhecimento daquilo que é necessário e daquilo que 0 indivíduo crê ser importante.

Uma revisão sistemática recente apresentou o empoderamento do paciente como promotor da adesão à farmacoterapia, desde que haja um senso de controle construído entre médico e paciente, onde as crenças dos pacientes em sua própria capacidade e controle sobre sua saúde estão presentes simultaneamente com seu reconhecimento do papel do médico no manejo da doença ${ }^{14}$. A autonomia e 0 empoderamento têm importância por criar condições, não só para que o indivíduo perceba seu estado de saúde, mas também para construir uma consciência ou sua própria visão de sua saúde.

Sobre as estatísticas de consumo de medicamentos, estudos revelaram que 0 gasto brasileiro em 2015 foi de $R \$ 92,5$ bilhões, cerca de $1,5 \%$ do Produto Interno Bruto $(\mathrm{PIB})$ daquele ano ${ }^{15}$. E ainda há uma estimativa de que o mercado global de produtos farmacêuticos chegue, em 2020, a US\$ 1,3 trilhão em comercialização e que - Brasil passará a ser o quinto desse mercado ${ }^{16}$.

Destacam-se, como um sério problema de saúde pública, as doenças crônicas não transmissíveis - DCNT (cardiovasculares, crônicas, diabetes mellitus, neoplasias, entre outras), que são resultado de determinantes sociais e condicionantes, além de fatores de risco individuais como tabagismo, consumo nocivo de álcool, inatividade física e alimentação não saudável ${ }^{17}$.

Em 2012, as DCNT foram as principais causas de morte no mundo, correspondendo a $68 \%$ dos óbitos. Aproximadamente $75 \%$ das mortes por DCNT ocorrem em países de baixa e média renda ${ }^{18}$. Devido ao tratamento farmacológico de longo prazo possibilitar o controle dessas doenças, torna-se 
fundamental a avaliação da adesão e o acompanhamento por profissionais da saúde.

Em uma perspectiva de modificar o atual cenário da TM e, portanto, da adesão terapêutica, utilizam-se as Tecnologias da Informação e Comunicação (TIC), as quais potencializam 0 desenvolvimento de estratégias favoráveis a uma educação de qualidade. As TIC englobam ferramentas, métodos e saberes na transmissão de conhecimento. Também participam do processo de ensino-aprendizagem quando acompanhados de métodos pedagógicos ${ }^{19}$.

Em busca da eficiência nos processos de trabalho em saúde e na qualidade do atendimento - além da redução de erros e de filas e do fortalecimento das ações de capacitação para o uso eficiente e eficaz das novas tecnologias - houve a necessidade de avançar no acesso à Internet e na disponibilidade de sistemas de informação de apoio ${ }^{14}$. A progressiva introdução das TIC em nosso dia-a-dia destaca as potencialidades que este tipo de ferramenta disponibiliza para facilitar, entre outras coisas, novas formas de comunicação e interação social.

A adesão ao tratamento, medicamentoso ou não, é fundamental para o sucesso da terapia instituída pelo médico e equipe de saúde ${ }^{20}$. Alguns estudos têm demonstrado o sucesso de determinadas intervenções na adesão ao tratamento.

Observou-se que acompanhamento e educação ao paciente durante consultas semanais ou mensais mostram efeito positivo sobre a adesão ao tratamento ${ }^{21}$.

O profissional farmacêutico exerce uma atividade de elevada importância ao oferecer informações, esclarecer dúvidas, acompanhar o tratamento e ao tomar decisões compartilhadas juntamente com o paciente, promovendo o autocuidado e a autonomia deste ao estimular uma visão própria de sua condição de saúde.

Alguns fatores podem interferir diretamente na adesão a um tratamento medicamentoso, tais como: aceitação pelo paciente, necessidades particulares do paciente, custo do tratamento, acesso ao medicamento, complexidade de esquema terapêutico, presença de efeitos colaterais, ausência de sintomas, compreensão da doença ou problema de saúde, acompanhamento profissional, abordagem pelo profissional, relação profissionalpaciente e autonomia do paciente ${ }^{22}$.

Entendido como a necessidade do paciente de receber 0 medicamento apropriado, na dose correta, por adequado período de tempo, a baixo custo para ele e a comunidade, o uso racional de medicamentos (URM) é uma prática essencial para se obter a adesão do paciente à farmacoterapia ${ }^{23}$.

Embora sem consenso, entende-se a adesão a medicamentos como a utilização dos medicamentos prescritos em pelo menos $80 \%$ de seu total, observando horários, doses e tempo de tratamento. 
Pacientes com uso inferior a $80 \%$ apresentam risco quatro vezes maior de apresentarem eventos cardiovasculares agudos. Vários métodos têm sido utilizados na avaliação da baixa adesão, dentre eles os questionários e registros de dispensação de medicamentos ${ }^{24}$.

A importância e a complexidade que envolvem a adesão à farmacoterapia, a educação dos profissionais de saúde para que atuem na investigação dos motivos e fatores relacionados à adesão dos pacientes ao tratamento farmacológico, por meio de ambientes virtuais de aprendizagem, baseando-se na proposta da reflexão crítica da educação permanente em saúde e na construção compartilhada do conhecimento como modelo de ensino-aprendizagem fundamentam 0 presente estudo, contribuindo na autonomia e autoconhecimento do paciente, além de promover sua saúde.

Os objetivos deste estudo foram: desenvolver um curso para qualificar os profissionais de saúde na promoção da adesão dos pacientes à farmacoterapia por intermédio de um ambiente virtual de aprendizagem, avaliar as considerações dos participantes, apresentar ações de intervenção para resolução de problemas de adesão à farmacoterapia e disponibilizar o produto em acervos educacionais.

\section{Métodos}

O estudo, prospectivo e descritivo, desenvolveu um curso de qualificação para profissionais de saúde, hospedado na plataforma educacional Moodlecloud e que utiliza proposta educacional sócioconstrutivista para tratar o tema e a abordagem da estratégia da educação permanente em saúde, para estimular a reflexão crítica sobre o processo de trabalho em saúde. A avaliação do curso (facilidade no acesso, disposição visual, atividades de leitura, acesso a vídeos, fóruns e links, tutoria e informações) foi realizada a partir da participação voluntária de alunos do Programa de Pós-Graduação em Ensino na Saúde da Universidade Federal do Rio Grande do Sul. O período de avaliação do curso "Adesão à Farmacoterapia" foi de 25 de fevereiro a 15 de abril de 2018.

O curso está disponível no endereço eletrônico

https://pesquisaemsaude.moodlecloud.co $\mathrm{m} /$ login/index.php, mediante convite para acesso (Figura 1 - nos anexos).

Foram reunidos conteúdos e informações sob o formato de unidades de estudo. Fizeram parte do curso os seguintes tópicos: Unidade Um - O que é saúde (reflexões e atividades sobre 0 conceito), Unidade Dois - Determinantes sociais da saúde (discussões), Unidade Três - O que é medicamento (conceitos e debates), Unidade Quatro - O que é adesão (introdução ao assunto), Unidade 
Cinco - Fatores que influenciam na adesão (debates e contribuição dos participantes) e Unidade Seis - Ações centradas no paciente (sugestões para as práticas profissionais). Atividades como fóruns, discussão de casos, simulação de situações, produção textual foram inseridas nas unidades de estudo para estimular a interação entre os participantes do curso.

Para a avaliação do curso, foi utilizado um instrumento de autoavaliação pré-curso (Tabela 1), para conhecer o entendimento dos convidados a respeito dos assuntos abordados. Este instrumento foi composto por seis questões objetivas, tendo como opções de resposta números inteiros de zero a cinco, do tipo escala Likert, que referiu zero como o desconhecimento ou discordância e cinco como o conhecimento pleno ou concordância com o questionamento apresentado, e ainda a possibilidade de resposta pela opção 'NO' quando o participante não quis opinar. Outro instrumento utilizado, constituindo-se de 11 questões objetivas (com a mesma escala de avaliação) e uma questão descritiva, foi a avaliação pós-curso.

O estudo foi cadastrado na Plataforma Brasil e aprovado pelo Comitê de Ética em Pesquisa da Universidade Federal do Rio Grande do Sul sob o Certificado de Apresentação para Apreciação Ética (CAAE) no 80317117.0.0000.5347.

\section{Resultados}

Dos alunos convidados, sete responderam à autoavaliação pré-curso e nove à avaliação pós-curso. Sobre esta autoavaliação inicial, os resultados sugeriram que os participantes tinham pleno conhecimento sobre saúde e seus fatores determinantes, consciência sobre a importância da adesão à farmacoterapia, noção geral sobre adesão e fatores que a influenciam, mas pouco conhecimento sobre como agir profissionalmente na abordagem do tema adesão à farmacoterapia junto ao paciente.

Devido ao número de participantes ter sido pequeno, acreditou-se que uma análise quantitativa pelo número de acessos, pelas discussões nos fóruns e pelo desenvolvimento de atividades não teria representatividade. Portanto, foi realizada uma análise qualitativa referente às respostas recolhidas a partir do instrumento de autoavaliação pós-curso, no qual cada participante expôs suas percepções pessoais e profissionais sobre a experiência.

Após a análise do curso, os participantes realizaram a avaliação póscurso. Foram 11 perguntas, tendo como possibilidades de resposta números inteiros de zero a cinco, do tipo escala Likert (Tabela 2). Como resultados positivos informados pelos participantes, destacam-se a facilidade de acesso ao curso e o bom desempenho do pesquisador como tutor. Levando em 
consideração as respostas dos participantes, o visual, a organização, as informações, o material didático e o tempo de execução das atividades necessitam ser revisados e aperfeiçoados.

A última questão, que solicitava a opinião de cada participante, tinha a seguinte redação: 'Utilizando suas palavras, por favor, faça sua avaliação do curso apontando críticas, sugestões e/ou alterações. Avalie, critique e sugira o que 0 curso disponibilizou e o que pode ser alterado'.

Em relação ao tema e conteúdo, o curso continha leituras acessíveis a profissionais de diversas áreas. Sobre a forma de apresentação do curso, os participantes sugeriram definição de prazos para iniciar e finalizar as unidades de estudo e cada tarefa. Também foi sugerido um tempo maior entre os módulos, referindo-se às características do público-alvo (profissionais e mestrandos). Ausência de informações em relação ao andamento do curso (inserção de elementos gráficos) e aos temas que seriam abordados já na tela inicial, além de mais recursos audiovisuais, também foram sugestões recebidas.

Complementando a questão visual do curso, foram sugeridas atividades audiovisuais, para que o curso equilibrasse a proporção entre arquivos de textos e mídias.

As atividades assíncronas do curso, aquelas que não necessitam da participação simultâneas dos envolvidos, permitem aos alunos a definição de seu ritmo de trabalho sem que a interação seja prejudicada, desde que as atividades incentivem o pensamento crítico e a reflexão. Apropria-se dos benefícios do ensino a distância ao possibilitar a ampliação do acesso à educação, o aperfeiçoamento da qualidade, da eficácia, da motivação e da eficiência dos processos de ensino e de aprendizagem, por meio do estímulo à melhoria das relações interpessoais entre os discentes e docentes, bem como 0 fomento à autonomia intelectual discente e à reflexão continuada sobre a atuação pedagógica docente. Acrescenta-se também como vantagens a diminuição dos custos para o desenvolvimento de cursos nessa modalidade e a possibilidade de atendimento individualizado.

Foi observado que, além da definição dos objetivos, as oportunidades de construção do conhecimento, a existência de espaços de expressão individual e coletiva, a possibilidade de oferecer recursos digitais em diferentes formatos e as facilidades no gerenciamento das atividades e na avaliação foram fundamentais para 0 alcance dos objetivos propostos.

Apesar de a proposta do curso não ser formativa, ele estimula a consciência dos profissionais de saúde a atuar promovendo o autocuidado dos pacientes em relação ao uso de medicamentos. Também reforça que uma das formas mais efetivas de melhorar o uso de 
medicamentos é a educação dos profissionais de saúde, pois o uso incorreto de medicamentos deve-se comumente a polifarmácia, uso indiscriminado de antibióticos, prescrição não orientada por diretrizes, automedicação inapropriada e o grande incentivo terapêutico disponibilizado comercialmente.

A importância de oportunizar um curso para os profissionais da área da saúde de diversas formações é relevante para que a equipe profissional se comunique entre si e com os usuários de medicamentos, compreendendo assim suas dificuldades, esclarecendo suas dúvidas e atendendo suas expectativas, permitindo que entendam a relação da saúde/doença com a medicação. A Figura 2 apresenta algumas atividades do curso disponibilizadas na plataforma Moodlecloud.

O URM resulta de relações entre diferentes atores sociais, evidenciando as responsabilidades do governo, dos profissionais de saúde e da sociedade para a sua efetivação. Isso quer dizer, no universo complexo, que a promoção do URM envolve iniciativas, nível de organização política e social e também o conhecimento da representação simbólica do que é medicamento na sociedade ${ }^{25}$.

O curso de Adesão à Farmacoterapia propôs conscientizar os profissionais de saúde a promover o autocuidado junto aos pacientes, estimulando assim a adesão ao tratamento, redução dos desperdícios, prevenção de erros com medicamentos, interações medicamentosas e reações adversas e destinação adequada dos resíduos. Essas ações devem estar integradas às rotinas dos serviços de saúde e ao trabalho da equipe de saúde como um todo.

Como principal desafio da continuidade do curso e do ensino ofertado na modalidade a distância está a evasão. Falta de motivação, integração social, suporte tecnológico, interesses pessoais e métodos didático-pedagógicos foram alguns dos motivos citados pelos autores, não sendo identificada por eles a predominância de algum em particular. Para este estudo, a evasão também foi identificada sendo motivada possivelmente por falta de informações detalhadas e questões pessoais.

\section{Conclusão}

Os textos, as atividades e outros materiais componentes do curso foram dispostos de modo a estimular a discussão e a opinião dos participantes. A possibilidade da troca de experiências intermediada por tutoria permite 0 desenvolvimento do conhecimento, qualificando o profissional de saúde para promover a adesão dos pacientes à farmacoterapia.

O curso oferece sugestões de ações de intervenção junto ao paciente. Dentre os métodos apresentados estão o calendário posológico, aplicativos para 
celular, etiquetas com figuras e informações, porta-comprimidos e dispensadores eletrônicos.

Como propõe o curso de Adesão à Farmacoterapia, a adesão pode e deve ser estimulada por todos os profissionais de saúde. O empenho multiprofissional, além de valorizar 0 trabalho de cada profissional, também assegura a integralidade da atenção à saúde. Alguns métodos e ações para avaliar e incentivar a adesão e o uso correto de medicamentos foram apresentados com o intuito de que os profissionais de saúde os acrescentem em suas práticas.

As atividades reflexivas e colaborativas apresentadas no curso empenharam-se em utilizar a problematização como método pedagógico, já praticado nas equipes de saúde de atenção primária. Buscou-se a partir daí 0 desenvolvimento de conhecimentos que relacionam a teoria e a prática.

No presente estudo, o produto (curso) desenvolvido ao longo deste mestrado profissional, assim como as ações e serviços de saúde, destinam-se à manutenção das condições de saúde e ao estímulo do autocuidado e autonomia do paciente em relação a si próprio. Para isso, percebe-se a necessidade de estabelecer o vínculo entre profissional e paciente, de levar em consideração seus saberes, de reconhecer que a saúde é condicionada por diversos fatores - e que a adesão representa um desses fatores - e, principalmente, da postura dos profissionais em estarem dispostos a refletir, a participar, a exercitar de forma permanente $o$ processo de ensinar e aprender, a desfazer e refazer o conhecimento, para que o saber e o fazer se somem com o objetivo de proporcionar condições para um satisfatório desenvolvimento, individual e coletivo, de autonomia, reflexão e igualdade.

\section{Referências}

1. Luiza VL, Tavares NUL, Oliveira MA, Arrais PSD, Ramos LR, Dal Pizzol TS, et al. Gasto catastrófico com medicamentos no Brasil. Revista de Saúde Pública [Internet]. 2016 [citado em 23 de Maio de 2017], 50(supl 2): 1-9. Disponível em: http://www.scielo.br/pdf/rsp/v50s2/pt_0034 -8910-rsp-s2-S01518-

87872016050006172.pdf

2. Laffin NHF, Bonacim CAG. Custos da saúde: judicialização de medicamentos ofertados pelo SUS. XXIV Congresso Brasileiro de Custos [Internet]. Novembro de 2017 [citado em 09 Abril de 2019], anais. Disponível em:

https://anaiscbc.emnuvens.com.br/anais/a rticle/viewFile/4312/4312

3. Arrais PSD, Fernandes MEP, Dal Pizzol TS, Ramos LR, Mengue SR, Luiza VL. Prevalência da automedicação no Brasil e fatores associados. Rev Saúde Pública [Internet]. 2016 [citado em 09 de Abril de 2019], 50(supl 2):13s. Disponível em: http://www.rsp.fsp.usp.br/wpcontent/uploads/articles_xml/0034-8910rsp-s2-S0151887872016050006117/0034-8910-rsp-s2S01518-87872016050006117pt.x83745.pdf

4. Remondi FA, Oda S, Cabrera MAS. Não adesão à terapia medicamentosa: da teoria à prática clínica. Revista de Ciências Farmacêuticas Básica e 
Aplicada. Abril de 2014 [citado em 24 de Maio de 2017], 35(2): 177-185. Disponível em: <http://serv-

bib.fcfar.unesp.br/seer/index.php/Cien_Fa rm/article/viewFile/2836/2836> Acesso em: 24 mai 2017.

5. Brasil. Síntese de evidências para políticas de saúde: adesão ao tratamento medicamentoso por pacientes portadores de doenças crônicas. Ministério da Saúde [Internet]. 2016 [citado em 09 de Abril de 2019]. Disponível em:

http://bvsms.saude.gov.br/bvs/publicacoes /sintese_evidencias_politicas_tratamento_ medicamentoso.pdf

6. Mathias TL, Guidoni CM, Girotto E. Tendências de casos de envenenamento relacionados a drogas atendidos em um centro de controle de intoxicação. Rev Bras Epidemiol. Abril de 2019 [citado em 10 de Abril de 2019], 22: e190018. doi: 10.1590 / 1980-549720190018.

7. Berreni A, Montastruc F, BondonGuitton E, Rousseau V, Abadie D, Durrieu $G$, et al. Adverse drug reactions to selfmedication: a study in a pharmacovigilance database. Fundamental \& Clinical Farmacology [Internet]. Outubro de 2015 [citado em 03 de Outubro de 2018], 29(5):517-20. Disponível em:

https://www.ncbi.nlm.nih.gov/pubmed/2621 5671

8. Ruiz ME. Risks of self-medication practices. Current Drug Safety [Internet]. Outubro de 2010 [citado em 04 de Outubro de 2018], 5(4):315-23. Disponível em:

https://www.ncbi.nlm.nih.gov/pubmed/206 15179

9. Fettah $\mathrm{H}$, Moutaouakkil $Y$, Sefrioui MR, Moukafih B, Bousliman Y, Bennana A, et al. Detection and analysis of drug-drug interactions among hospitalized cardiac patients in the Mohammed V Military Teaching Hospital in Morocco. Pan African Medical Journal [Internet]. Abril de 2018 [citado em 03 de Outubro de 2018], 29(225). Disponível em: http://www.panafrican-med- journal.com/content/article/29/225/pdf/225 .pdf

10. Haghighi S, Ashrafzadeh H, Sayadi N. Self-medication and related factors among university students in Iran. Journal of Nursing and Midwifery Sciences. 2016; 3(2): 47-51.

11. Dean Carlos Nascimento de Moura DCN, José Reginaldo Pinto JR, Martins $P$, Pedrosa KA, Carneiro MGD. Uso abusivo de psicotrópicos pela demanda da estratégia saúde da família: revisão integrativa da literatura. Sanare.

Dezembro de 2016 [citado em 10 de Abril de 2019], 15(2): 136-144.

12. World Health Organization. Adherence to Long-term Therapies: evidence for action [Internet]. Janeiro de 2003 [citado em 28 de Maio de 2017], 209p. Disponível em:

http://apps.who.int/iris/bitstream/10665/42 682/1/9241545992.pdf

13. Brasil. Ministério da Saúde.

Secretaria-Executiva. Secretaria de Vigilância em Saúde. Glossário temático: promoção da saúde. Ministério da Saúde [Internet]. 2012 [citado em 05 de Setembro de 2018]. Disponível em: http://bvsms.saude.gov.br/bvs/publicacoes /glossario_promocao_saude_1ed.pdf

14. Náfrádi L, Nakamoto K, Schulz PJ. Is patient empowerment the key to promote adherence? A systematic review of the relationship between self-efficacy, health locus of control and medication adherence. PLoSONE. Outubro de 2017 [citado em 11 de dezembro de 2018], 12(10): 1-23. Disponível em: https://www.ncbi.nlm.nih.gov/pmc/articles/ PMC5645121/pdf/pone.0186458.pdf

15. Oliveira N. Gastos com saúde crescem mesmo em meio à crise $\mathrm{e}$ atingem 9,1\% do PIB. Agência Brasil. Dezembro de 2017 [citado em 29 de Agosto de 2018]. Disponível em: http://agenciabrasil.ebc.com.br/economia/ noticia/2017-12/gastos-com-saudecrescem-mesmo-em-meio-crise-eatingem-91-do-pib

16. Wannmacher L. Importância dos 
medicamentos essenciais em prescrição e gestão racionais. Uso racional de medicamentos: temas selecionados. Ministério da Saúde. 2010 [citado em 01 de Setembro de 2018]. Disponível em: https://www.paho.org/bra/index.php?optio $\mathrm{n}=\mathrm{com}$ _docman\&view=download\&alias $=1$ 314-importancia-dos-medicamentosessenciais-em-prescricao-e-gestaoracionais-uso-racional-medicamentostemas-selecionados-n-2-

$4 \&$ category_slug=assistenciafarmaceutica-958\&ltemid=965

17. Brasil. Secretaria de Vigilância em Saúde. Plano de Ações Estratégicas para - Enfrentamento das Doenças Crônicas Não Transmissíveis (DCNT) no Brasil 2011-2022. Ministério da Saúde [Internet], 2011 [citado em 27 de Junho de 2017]. Disponível em:

http://bvsms.saude.gov.br/bvs/publicacoes /plano_acoes_enfrent_dcnt_2011.pdf 18. Tavares NUL, Costa KS, Mengue SS, Vieira MLFP, Malta DC, Silva Jr., JB. Uso de medicamentos para tratamento de doenças crônicas não transmissíveis no Brasil: resultados da Pesquisa Nacional de Saúde, 2013. Epidemiol. Serv. Saúde [Internet], Junho de 2015 (citado em 15 de Abril de 2019), 24(2): 315-323. Disponível em:

http://scielo.iec.gov.br/pdf/ess/v24n2/v24n 2a14.pdf

19. Rocha JSY. Uso de tecnologias da informação e comunicação na educação em saúde. Problematização e desenvolvimento. Medicina (Ribeirão Preto) [Internet]. 2015 [citado em 11 Julho de 2017], 48(3): 214-23. Disponível: http://dx.doi.org/10.11606/issn.21767262.v48i3p214-223

20. Centro Regional De Estudos Para O Desenvolvimento Da Sociedade Da Informação. TIC no setor de Saúde: disponibilidade e uso das tecnologias de informação e comunicação em estabelecimentos de saúde brasileiros. Panorama Setorial da Internet [Internet]. Janeiro de 2014 [citado em 05 de Outubro de 2018], 6(1): 1-9. Disponível em: https://cetic.br/media/docs/publicacoes/6/ Panorama_Setorial6.pdf
21. Gusmão JL, Mion Jr D. Adesão ao tratamento - conceitos. Revista Brasileira de Hipertensão [Internet]. 2006 [citado em 28 de Maio de 2017], 13(1): 23-25.

Disponível em: http://departamentos.cardiol.br/dha/revista /13-1/06-adesao-ao-tratamento.pdf

22. Van Wijk BL, Klungel OH, Heerdink $E R$, de Boer $A$, et al. Effectiveness of interventions by community pharmacists to improve patient adherence to chronic medication: a systematic review. Ann Pharmacother [Internet]. Fevereiro de 2005 [citado em 03 de Dezembro de 2018], 39(2): 319-28. Disponível em: https://www.ncbi.nlm.nih.gov/pubmed/156 32223

23. Brasil. Ministério da Saúde. Secretaria de Ciência, Tecnologia e Insumos Estratégicos. Uso racional de medicamentos: temas selecionados. Ministério da Saúde [Internet]. 2012 [citado em 16 de Agosto de 2018]. Disponível em:

http://bvsms.saude.gov.br/bvs/publicacoes /uso_racional_medicamentos_temas_sele cionados.pdf

24. Ben AJ, Neumann CR, Mengue SS. Teste de MoriskyGreen e Brief Medication Questionnaire para avaliar adesão a medicamentos. Rev. Saúde Pública [Internet]. Abril de 2012 [citado em 12 de julho de 2017], 46(2): 279-289. Disponível em:

http://www.scielo.br/pdf/rsp/2012nahead/3 357.pdf

25. Monteiro ER, Lacerda JT. Promoção do uso racional de medicamentos: uma proposta de modelo avaliativo da gestão municipal. Saúde Debate [Internet]. Dezembro de 2016 [citado em 10 de Abril de 2019], 40(111): 101-116. Disponível em:

http://www.scielo.br/pdf/sdeb/v40n111/010 3-1104-sdeb-40-111-0101.pdf 


\section{Anexos}

\section{Lista de figuras}

Figura 1 - Tela inicial do site Pesquisa em Saúde, da plataforma Moodlecloud.

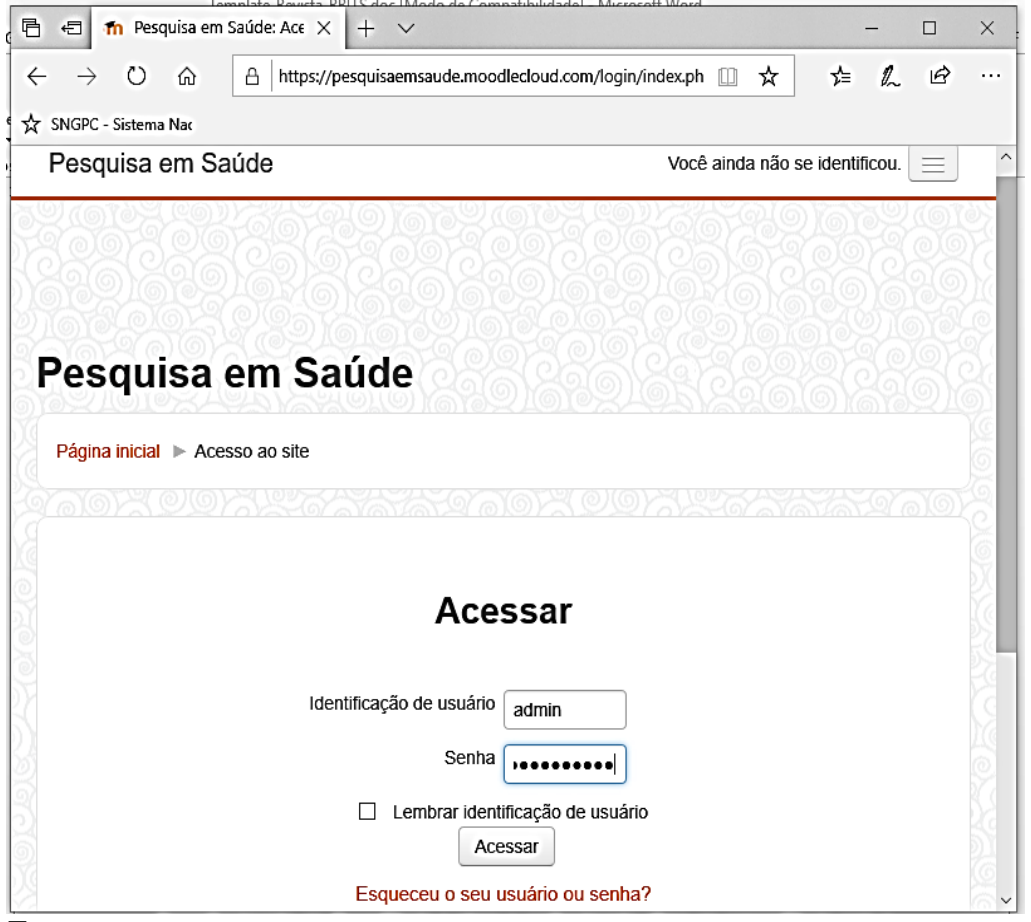

Fonte: os autores.

Figura 2 - Tela contendo atividades do curso Adesão à Farmacoterapia, na plataforma Moodlecloud.

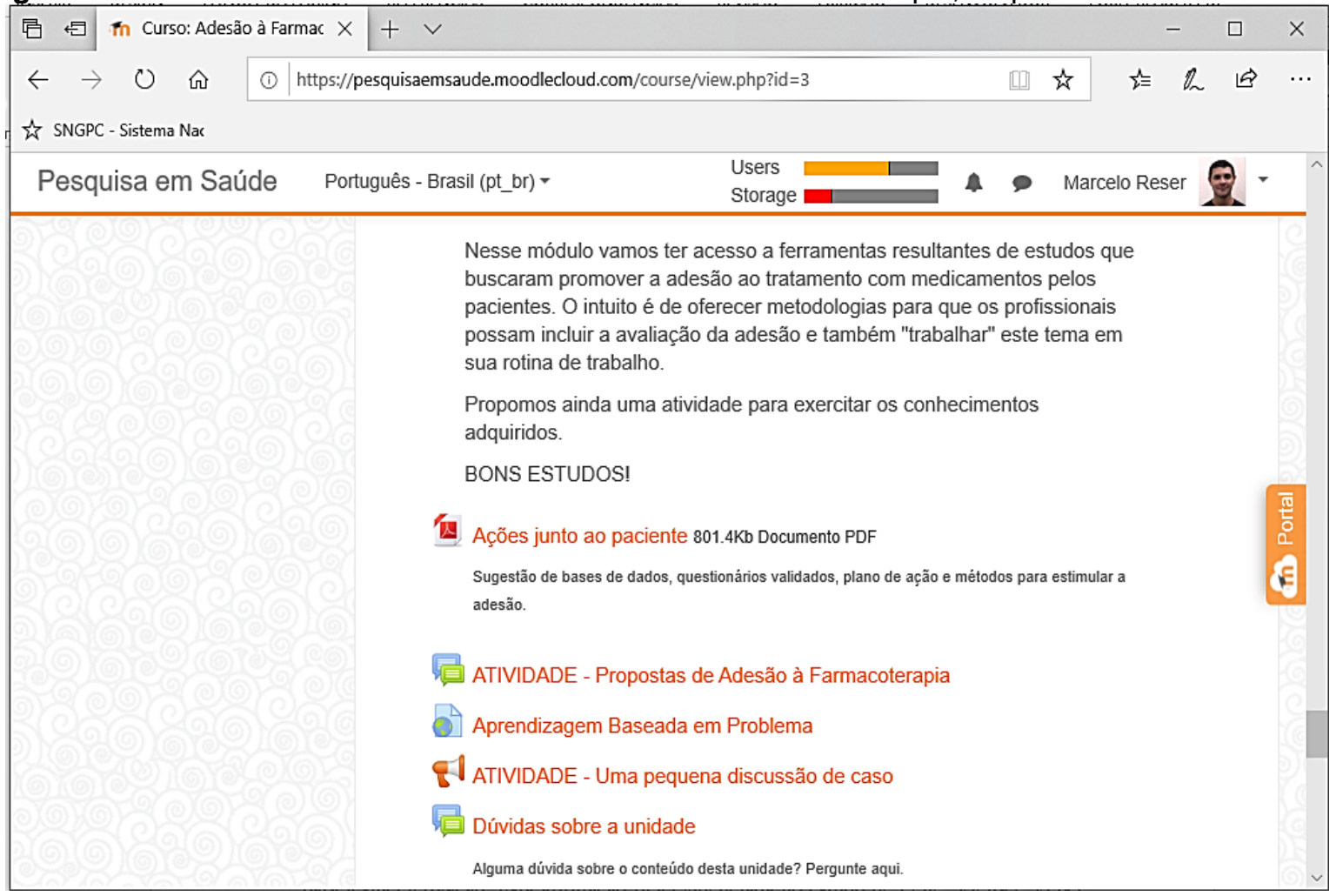

Fonte: os autores.

RE. SAÚD. DIGI. TEC. EDU., Fortaleza, CE, v. 4, n. 1, p. 60-73, jan./ago. 2019.

ISSN: 2525-9563 


\section{Lista de Tabelas}

Tabela 1 - Instrumento de avaliação pré-curso

1. Qual seu nível de entendimento sobre conceitos e fatores condicionantes e determinantes de saúde?

2. Qual seu nível de entendimento sobre o que é medicamento?

3. Qual seu nível de entendimento sobre o tema de adesão à terapia com medicamentos?

4. Qual seu nível de conhecimento sobre o que influencia a adesão à farmacoterapia?

5. Qual seu nível de conhecimento sobre métodos de abordagem do tema de adesão junto ao paciente ou público alvo?

6. Qual a importância que você dá ao tema proposto pelo curso em suas práticas profissionais?

${ }^{*}$ Respostas em números inteiros de 0 a 5 (Likert), sendo $0=$ discordância total e 5 = concordância total.

${ }^{* *} \mathrm{NO}=$ participante prefere não opinar.

Tabela 2 - Instrumento de autoavaliação pós-curso

1. O acesso à plataforma MoodleCloud e ao curso foi fácil?

2. O visual e a organização do curso no ambiente virtual são adequados?

3. A linguagem, as informações e o formato do material didático são satisfatórios para desenvolver o tema proposto?

4. Você acredita que o curso proporciona atividades que estimulem a interação e discussão entre participantes?

5. O tempo para a execução das atividades é adequado?

6. O curso atinge o objetivo proposto?

7. Você concorda que o curso possa ser dirigido a profissionais de diferentes áreas da formação acadêmica?

8. Você acredita que cursos a distância, utilizando ambientes virtuais de aprendizagem, são uma boa forma de se obter conhecimento?

9. O curso contribuiu para a aquisição de novos conhecimentos?

10. Você faria outros cursos na modalidade de ensino a distância?

11. O tutor (pesquisador) demonstrou clareza, objetividade, conhecimento sobre o assunto e habilidade na utilização deste ambiente virtual de aprendizagem?

${ }^{*}$ Respostas em números inteiros de 0 a 5 (Likert), sendo 0 = discordância total e 5 = concordância total.

${ }^{* *} N O=$ participante prefere não opinar.

RE. SAÚD. DIGI. TEC. EDU., Fortaleza, CE, v. 4, n. 1, p. 60-73, jan./ago. 2019.

ISSN: 2525-9563 


\section{Como citar este artigo}

Reser MR, Silva CHda. Plataforma Moodlecloud na qualificação de profissionais de saúde no processo de adesão à Farmacoterapia. Revista de Saúde Digital e Tecnologias Educacionais. [online], volume 4, n. 1. Editor responsável: Luiz Roberto de Oliveira. Fortaleza, mês e ano, p. 60-73. Disponível em: http://periodicos.ufc.br/resdite/index. Acesso em "dia/mês/ano".

Data de recebimento do artigo: 01/04/2019

Data de aprovação do artigo: 06/06/2019 